\title{
Adaptive S-ALOHA CDMA as an Alternative Way of Integrating Services in Mobile Environments
}

\author{
Oriol Sallent, Member, IEEE, and Ramon Agustí, Member, IEEE
}

\begin{abstract}
Code-division multiple-access (CDMA) schemes appear to be very promising access techniques for coping with the requirements of third-generation mobile systems, mainly because of their flexibility. This paper proposes an adaptive S-ALOHA DS-CDMA access scheme as a method for integrating nonreal-time (i.e., Internet applications) and real-time (i.e., voice) services in a multicell scenario by exploiting the potentials of CDMA under time-varying channel load conditions. The adaptive component makes data terminals autonomously change their transmission rate according to the total (voice+data) channel occupancy, so that the minimum possible data delay, which can be analytically obtained by defining a birth-death process, is almost always achieved. Moreover, by means of a simplified cellular model, the proposed algorithm revealed the same behavior, i.e., it tries to select the most suitable transmission rate at any time slot, when it is affected by intercell interference and even by power control imperfections. Finally, in order to gain more insight into the potentials of such an access strategy, the adaptive S-ALOHA CDMA scheme is then compared to a reservation time-division multiple-access (TDMA)-based protocol (PRMA++), showing the benefits of the CDMA-based solution in terms of capacity, flexibility, and data delay performance.
\end{abstract}

Index Terms-CDMA, mobile communications, multiple-access protocols.

\section{INTRODUCTION}

$\mathbf{T}$ HE ASTONISHING growth rates of the Internet and worldwide web (WWW) markets in recent years show the enormous potentials of packet-based services. Data traffic figures predicted for third-generation-on mobile communications that might have seemed far too high a few years ago are now seen as low. However, many of the packet schemes proposed for the third generation are still mainly voice-oriented schemes, especially time-division multiple-access (TDMA)-based schemes like PRMA [1] or PRMA++ [2], [3]. Nevertheless, in general, the bursty characteristics of data traffic should yield benefits for access technologies capable of allocating a large amount of simultaneous resources instantly and in a decentralized way, so as to reduce average delay and increase throughput. ALOHA-like techniques, largely used in LAN scenarios, could fulfill these requirements.

Radio resource allocation is also an important issue when designing future cellular systems [4]. By allowing any cell to allocate any resource, dynamic channel allocation (DCA) is

\footnotetext{
Manuscript received February 17, 1998; revised January 27, 1999. This work was carried out under the CICYT TIC94-0870-C02-01 Project within the framework of the National Plan of Spain.

The authors are with the Departament de Teoria del Senyal i Comunicacions, Universitat Politècnica de Catalunya, 1-08034 Barcelona, Spain (e-mail: oriol@xaloc.upc.es; ramon@xaloc.upc.es).

Publisher Item Identifier S 0018-9545(00)03697-5.
}

attractive because it does not need any cellular planning. DCA can use different schemes. One way is to allocate resources exposed to the least interference, as in DECT [5]. Unfortunately, the fast-changing interference environment appearing with bursty packet data prevents the use of least-interference strategies, since interference measurement periods can be similar to, or even higher than, a message transmission. Another way is to choose at random within the free resources. ALOHA-like access techniques could be included in this category and could be retained as appropriate for these scenarios. However, problems such as the high peak values associated with this access when moderate or high bit rates are employed [6], the unfair access results for nonfavored users when capture is introduced, and the inability to take advantage of full channel capacity when operating below saturation [7] all mean that ALOHA-like techniques are probably not the most suitable.

However, code-division multiple access (CDMA) jointly used with S-ALOHA [8], [9] seems a very promising way to deal with the increasing packet-type demand of Internet-like services. Since the multiple-access protocol remains the same, S-ALOHA CDMA schemes could retain the advantages of S-ALOHA. Moreover, the spectrum expansion introduced by this multiple-access technique (CDMA) avoids without the negative effects mentioned above the high-peak transmitted power necessary in a high-bandwidth S-ALOHA system, where, for the same bandwidth occupancy as in the S-ALOHA CDMA case, the time slot would be much shorter. In addition, CDMA can be considered a random resource allocation scheme and can also provide real-time services, like voice, with the quality of service (QoS) required. Moreover, the resulting S-ALOHA-CDMA would enable different services to be integrated easily, using natural statistical traffic multiplexing. This property is highly desirable in third-generation systems, since the constant evolution of the applications required generates uncertainty about what these systems will be required for: the scheme will be flexible, whatever the final solution.

Earlier studies of packet CDMA were initially limited to interactive data source models [9], [10]. On the basis of IS-95, intensive work was done on circuit-oriented CDMA for voice applications [11]. The need to integrate both types of traffic generated a lot of interest in this topic. Thus, [12] focused on the effect of FEC and the propagation loss coefficient on network capacity. Approaches considered in [8], [13], and [14] deal with receiver-oriented systems, where subscribers have to share a reduced set of code sequences. However, although all of these papers on packet CDMA show the flexibility inherent to CDMA, none of them fully exploits the potentials of CDMA by taking full advantage of time-varying channel load conditions. 
This paper will consider an adaptive S-ALOHA CDMA access [15], [16] as a strategy for coping with the requirements of both bursty packet and voice transmission operating in a multicell scenario. In addition, this technique, which can be decentralized (mobile-controlled), allows us to use the current available capacity of the channel, which varies with the traffic load, so as to decrease the message delay delivery when the traffic load decreases. For this purpose, we take advantage of the CDMA's flexibility in bandwidth utilization, as it allows the change in processing gain. In fact, there are scenarios that are not capacity limited, i.e., those with reduced traffic loads, as in many indoor environments, where S-ALOHA is optimum because the channel access delay is almost zero and the system is much simpler than other schemes.

The objective of this paper is to exploit the flexibility that the adaptive S-ALOHA CDMA access scheme provides in a multicell scenario, with moderate traffic needs for basically nonreal-time services (i.e., WWW) and with the capacity to integrate real-time services (i.e., voice). In particular, the main results of this paper are as follows: 1) to demonstrate that the proposed access scheme is able to optimize the bandwidth allocation in a service integrated system; 2) to validate that the proposed scheme behaves well in the presence of intercell interference and also of power control imperfections; and 3) to show that the claimed advantages of the proposed scheme compares favorably to PRMA++ in both complexity and performance. To this end, the claimed advantages of the noncentralized proposed scheme are also compared with those of the centralized PRMA++ in a simplified multicell model. This comparison is made on the basis of the same number of vocal users in both schemes.

The rest of the paper is organized as follows. In Section II, an analytical model for message transmission under S-ALOHA DS-CDMA is presented in terms of both throughput and delay performance. This model is modified in Section III in order to obtain the optimum behavior that could be achieved with an adaptive change of the transmission rate algorithm applied to the S-ALOHA DS-CDMA scheme. A practical algorithm is proposed in Section IV. Section V demonstrates that this solution is able to integrate voice and data terminals, reducing data transmission delay, while in Section VI it is shown that the resulting system again tries to choose the most suitable transmission rate when it is affected by intercell interference and imperfect power control. Section VII compares the proposed scheme with a reservation protocol (PRMA++), pointing out the advantages in terms of flexibility and performance of the adaptive S-ALOHA-CDMA solution. Finally, some conclusions close the paper in Section VIII.

\section{S-ALOHA DS-CDMA MODEL}

A BPSK DS-CDMA access system with a processing gain given by $G_{p}$ is discussed. Under a transmitter-oriented approach, all users are assigned their own random PN signature sequence. The network topology consists of a multiple-receiver scheme at the central base station and a number of users located around it in line with the conventional star architecture that enables uplink and downlink transmission paths to exist on two different frequency bands. Additionally, a perfect power control capable of mitigating fadings of the channel is discussed, i.e., the terminal transmits the power level necessary to maintain the same received power at all times. Instantaneous power control permits a huge improvement in system performance over open-loop power control and is in fact taken into account in the already operative [11] and proposed [17], [18] CDMA systems.

If ideal instantaneous power control is assumed, the channel can be seen as an additive white Gaussian noise (AWGN) if we use the Gaussian hypothesis to model the interference caused by other users [19]. Even in a packet-access system, the Gaussian hypothesis should not greatly influence throughput behavior [20]. Under these conditions, the following expressions hold for the evaluation of the bit error rate (BER):

$$
P_{b}(n)=Q\left(\sqrt{2 \frac{E_{b}}{N_{o}}}\right), \quad \text { with } \quad \frac{E_{b}}{N_{o}}=\frac{3 G_{p}}{2(n-1)}
$$

where $n$ is the number of simultaneous users. Then, the probability of correctly detecting a packet containing $L \mathrm{~b}$ is

$$
P_{c}(n)=\left[1=P_{b}(n)\right]^{L}
$$

Keeping the transmission bandwidth constant and taking as reference a transmission rate of $v \mathrm{~b} / \mathrm{s}$, packet length of $L \mathrm{~b}$, and a processing gain $G_{p}$, the transmission rate could be increased by a factor $\alpha(\alpha>1)$ at the expense of a reduction in the spread capacity. That is, transmitting at rate $\alpha v$ b/s would allow a processing gain of $G_{p} / \alpha$. However, in exchange for this reduction in processing gain, $\alpha L$ b per time slot can be allocated, instead of only $L$.

When S-ALOHA DS-CDMA operates below the saturation point, not all the features are used efficiently. In particular, when the channel load is low, a high processing gain is not necessary because there is little interference in the system. One could say that it is the access technique that limits the throughput of the system under low loads instead of what one expects in a CDMA system, which should be interference limited. Since the need for processing gain (protection against multiuser interference) depends on the channel load, the system performance can be improved by using an adaptive change of the transmission rate algorithm according to the channel load conditions.

Although a multirate CDMA scheme will be discussed, let an analytical model for the system with a single transmission rate be introduced first. For this purpose, suppose that $N$ registered users generate messages with arrival rate $\lambda$ according to a Poisson process. Messages are exponentially distributed with average message length $(1 / u) \mathrm{b} /$ message. Since time is slotted and the length of the message may not coincide with the length of a packet, it must be completed by dummy bits if too small or it must be conveyed over several packets if too large. The first parameter that needs to be defined before performance can be measured is the average number of packet transmissions

$$
N(n)=\sum_{i=1}^{\infty} i\left[1-P_{c}(n)\right]^{i-1} P_{c}(n)=\frac{1}{P_{c}(n)}
$$




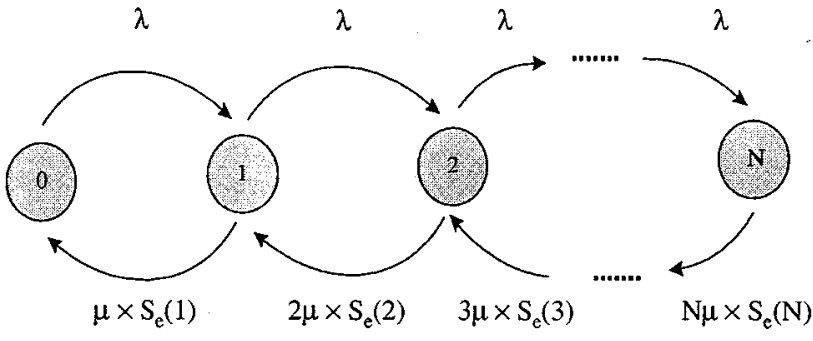

Fig. 1. S-ALOHA DS-CDMA states system model.

The effective channel utilization $S_{e}(n)$ is also defined

$$
S_{e}(n)=\frac{L}{N(n)} \text { bits/slot }
$$

where $L$ is the packet length expressed in bits. Equation (4) reveals the difference between the system-offered load and the channel-offered load, since the channel is useful only in one out of $N(n)$ slots.

The system model is shown in Fig. 1, where the states of the resulting birth-death process are the number of simultaneous users [21]. Transition from state $i$ to state $(i+1)$ is associated with a new message arrival, while the service rate is given by the effective channel utilization, taking into account that CDMA transmission has as many serving queues as simultaneous users.

Let $P(n)$ denote the distribution of the number of simultaneous users. The flow condition over a generic state can then be written as

$$
P(n) \times \lambda=(n+1) \times \mu \times S_{e}(n+1) \times P(n+1)
$$

for $0 \leq n<N$. These equations and the normalization condition

$$
\sum_{n=0}^{N} P(n)=1
$$

lead to a linear equation system whose solution gives the equilibrium distribution of simultaneous users. Once this distribution is known, average throughput becomes

$$
S=\sum_{n=0}^{N} n \times S_{e}(n) \times P(n) .
$$

At this point, we can introduce the average number of packet retransmissions $T(n)$ in order to obtain the average delay for a S-ALOHA CDMA packet. Thus

$$
T(n)=\sum_{i=1}^{\infty} i\left[1-P_{c}(n)\right]^{i} P_{c}(n)=\frac{1-P_{c}(n)}{P_{c}(n)}
$$

where a constant number of simultaneous users $n$ from the first packet transmission up to its successful reception is assumed. The resulting model is sufficiently accurate for our purposes, as we will show in Section III. Then

$$
D(n)=1+T(n) \times \frac{1}{p_{r}}
$$

indicating that the packet delay is unavoidably one-slot transmission time plus the additional delay caused by retransmissions. According to the S-ALOHA operation, retransmissions are attempted at any slot with probability $p_{r}$, so we have an average delay $\left(1 / p_{\boldsymbol{r}}\right)$ for every packet retransmission. Finally, the average message delay is given by

$$
D=\sum_{n=0}^{N} \frac{1 / \mu}{L} \times D(n) \times P(n) .
$$

\section{Optimum PeRformance for Message DATA TRANSMISSION}

The previous model can be modified in order to obtain the optimum throughput that can be achieved with a change of the transmission rate algorithm. Ideally, this algorithm should be able to change the transmission rate used by the mobiles as a function of the system traffic load, so that the maximum possible throughput can always be obtained. Without losing general applicability, we will only consider three different transmission rates in the sequel: $\alpha v \mathrm{~b} / \mathrm{s}$ with $\alpha=1,2$, or 4 . Then, this algorithm should be able to maximize the number of correctly received bits for a given packet duration period by selecting the most suitable mobile transmission rate $(4 v, 2 v, v \mathrm{~b} / \mathrm{s})$ at any given slot depending on the number of active users (i.e., the interference level).

To attain this optimum throughput, the best combination of transmission rates should be obtained provided $n$ simultaneous users are present. Actually, they follow after an exhaustive search according to

$$
\begin{array}{lc}
\max _{\left(n_{v}, n_{2 v}, n_{4 v}\right)} & S\left(n_{v}, n_{2 v}, n_{4 v}\right) \\
\text { subject to } & n_{v}+n_{2 v}+n_{4 v}=n
\end{array}
$$

where

$n_{v} \quad$ number of users transmitting at $v \mathrm{~b} / \mathrm{s}$;

$n_{2 v} \quad$ number using $2 v \mathrm{~b} / \mathrm{s}$;

$n_{4 v}$ number at $4 v \mathrm{~b} / \mathrm{s}$;

$n \quad$ total number of simultaneous users;

$S$ throughput in such a combination of transmission rates.

Once the optimum number of users transmitting at every rate $\left(n_{v}^{*}, n_{2 v}^{*}, n_{4 v}^{*}\right)$ is known for every $n$, we can proceed to define the average number of packet transmissions for every transmission rate as

$$
N_{\alpha v}(n)=\frac{1}{P_{c,(\alpha v)}\left(n_{v}^{*}, n_{2 v}^{*}, n_{4 v}^{*}\right)} \quad \alpha=1,2,4
$$

where $P_{c,(\alpha v)}\left(n_{v}^{*}, n_{2 v}^{*}, n_{4 v}^{*}\right)$ indicates that the packet success probability depends on the transmission rate used for those users transmitting at rate $\alpha v \mathrm{~b} / \mathrm{s}$. We note that the expressions used to evaluate the BER must be modified in order to take into account the fact that the channel will be shared by users with different rates, and, consequently, in order to keep the same received $E_{b}$ for all users, the power received for $v \mathrm{~b} / \mathrm{s}$ is half of that required for $2 v \mathrm{~b} / \mathrm{s}$ and a quarter of that needed with $4 v \mathrm{~b} / \mathrm{s}$. In general, considering $n_{\alpha v}$ users transmitting at $\alpha v \mathrm{~b} / \mathrm{s}(\alpha=1,2, \cdots, K)$ and $G_{p}$ being the processing gain achieved when transmitting 


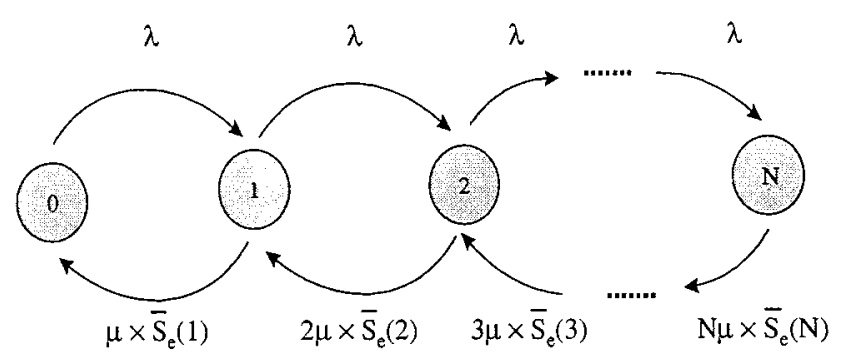

Fig. 2. Model for obtaining the optimum performance.

at $v \mathrm{~b} / \mathrm{s}(\alpha=1)$, the packet success probability under perfect power control can be calculated as

$$
\begin{gathered}
\left(\frac{E_{b}}{N_{o}}\right)_{\alpha v}=\frac{1}{\frac{2}{3 G_{p}}\left[\sum_{\substack{i=1 \\
i \neq \alpha}}^{K} i \times n_{i v}+\alpha \times\left(n_{\alpha v}-1\right)\right]} \\
P_{b,(\alpha v)}\left(n_{v}, n_{2 v}, \cdots, n_{K v}\right)=Q\left(\sqrt{2\left(\frac{E_{b}}{N_{o}}\right)_{\alpha v}}\right) \\
P_{c,(\alpha v)}\left(n_{v}, n_{2 v}, \cdots, n_{K v}\right) \\
=\left[1-P_{b,(\alpha v)}\left(n_{v}, n_{2 v}, \cdots, n_{K v}\right)\right]^{\alpha L} .
\end{gathered}
$$

We can also define the effective channel utilization for every transmission rate as

$$
S_{e,(\alpha v)}(n)=\frac{\alpha \times L}{N_{\alpha v}(n)} \quad \text { bits/slot }
$$

where the higher the rate, the higher the number of transported bits per packet. In the states system model in Fig. 2, an average channel utilization is introduced

$$
\begin{aligned}
\bar{S}_{e}(n)=\frac{1}{n}[ & n_{v}^{*} \times S_{e,(v)}(n)+n_{2 v}^{*} \times S_{e,(2 v)}(n) \\
& \left.+n_{4 v}^{*} \times S_{e,(4 v)}(n)\right] .
\end{aligned}
$$

This states system model enables the equilibrium distribution of simultaneous users to be obtained as in (5) by simply considering $\bar{S}_{e}(n)$ instead of $S_{e}(n)$. Thus, the optimum throughput is given by

$$
\begin{gathered}
S=\sum_{n=0}^{N}\left[n_{v}^{*} \times S_{e,(1 v)}(n)+n_{2 v}^{*} \times S_{e,(2 v)}(n)\right. \\
\left.+n_{4 v}^{*} \times S_{e,(4 v)}(n)\right] \times P(n) .
\end{gathered}
$$

The same procedure is also used for packet delay. Let us define the average packet transmission delay for every transmission rate

$$
D_{\alpha v}(n)=1+\left[\frac{1-P_{c,(\alpha v)}(n)}{P_{c,(\alpha v)}(n)}\right] \times \frac{1}{p_{r}}
$$

so that the average message delay is

$$
\begin{aligned}
D= & \sum_{n=1}^{N} \frac{1 / \mu}{L} \times \frac{1}{n} \times\left[n_{1 v}^{*} \times D_{1 v}(n)+\frac{1}{2} n_{2 v}^{*}\right. \\
& \left.\times D_{2 v}(n)+\frac{1}{4} n_{4 v}^{*} \times D_{4 v}(n)\right] \times P(n) .
\end{aligned}
$$

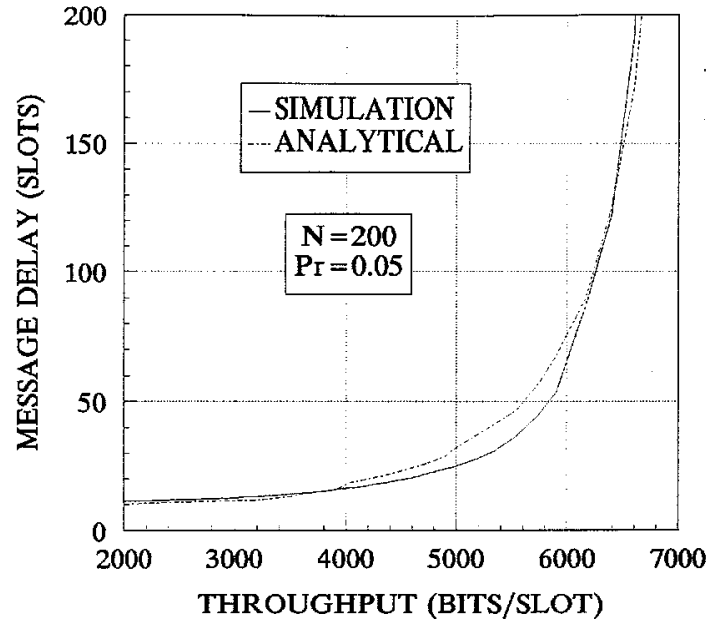

Fig. 3. Validation of the analytical model.

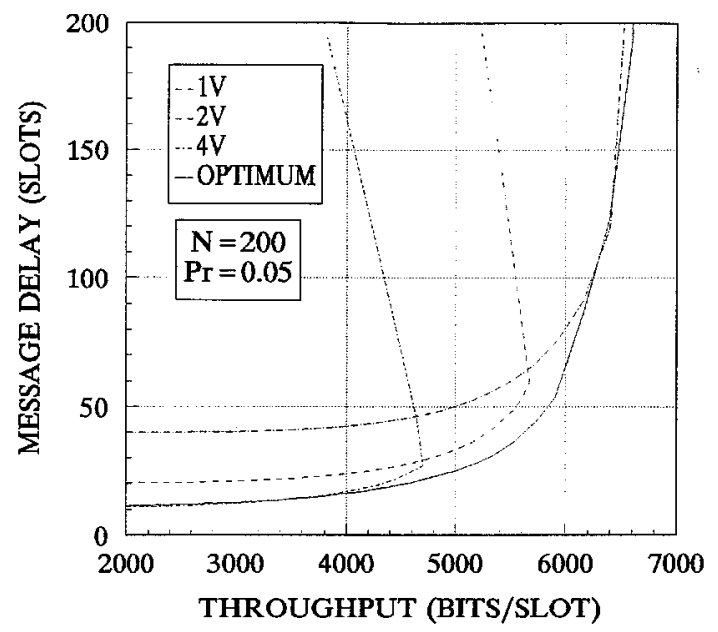

Fig. 4. Comparison of the optimum performance and the fixed transmission rates.

Fig. 3 compares the optimum throughput predicted analytically with the plot obtained by simulation. Results have been obtained for $N=200$ registered users, $G_{p}=127$ and $L=200$ for the reference transmission rate $(v \mathrm{~b} / \mathrm{s})$, a fixed retransmission probability $p_{r}=0.05$, average message length $(1 / \mu)=1 \mathrm{Kbyte}$, and $\lambda$ variable. Close agreement is found. Fig. 4 plots the throughput versus message delay performance for the three different rates and the optimum profile that could be achieved, which is the envelope of the fixed transmission rate performances. It can be seen from Fig. 4 that for low loads an asymptotic message delay of ten slots, corresponding to ten consecutive successful transmissions of $800 \mathrm{~b}$ per time slot, is attained at $4 v \mathrm{~b} / \mathrm{s}$. An asymptotic message delay of 20 time slots at $2 v \mathrm{~b} / \mathrm{s}$ and 40 time slots at $v \mathrm{~b} / \mathrm{s}$ shows the potential benefit of using an adaptive transmission rate procedure. We note that throughput figures are expressed in bits/slot, so that it is not necessary to fix the time slot duration and, consequently, results can be extended to any transmission rate.

\section{MS ALGORITHM}

In Sections II and III, we have pointed out the potentials of an adaptive change of the transmission rate algorithm. We now 
propose a practical solution. The proposed simple algorithm performed by the mobile station (MS) works as follows: each terminal traces its own evolution during the transmission time, i.e., terminals count their successful and erroneous packets. In the absence of errors, the mobile will assume a low traffic load and try to use a higher transmission rate. The throughput should be increased in this way. If errors occur, the mobile decides that the channel is overloaded and tries a lower transmission rate. In this case, fewer bits per packet are transmitted, but an overall improvement in throughput should follow, because these bits can be detected correctly since processing gain increases accordingly [15], [16]. So, such an access algorithm could be seen as a dynamic bandwidth allocation scheme.

Specifically, the MS needs to establish only two parameters: the number of consecutive packet failures before changing to a lower rate (max_tr) and the number of consecutive packet successes before trying a higher rate (min_suc). Therefore, the practical implementation of this algorithm would be very simple, since it only requires a counter in the mobile terminal and no spending on radio resources for signaling purposes. In fact, the MS algorithm performance can be easily improved with regard to the original proposal in [15] and [16] by simply allowing the values (max_tr, min_suc) to adaptively change instead of remaining fixed, thus avoiding "ping-pong" effects between two different transmission rates.

Fig. 5 shows the throughput attained with the MS algorithm. In spite of the simplicity of the algorithm, the envelope of the three individual graphs is almost reached, which is near optimum behavior (Fig. 4). Results were obtained with original values max_tr $=1$ and min_suc $=20$. Additionally, on one hand max_tr is increased by one every time that ten consecutive successes occur when transmitting at the highest rate. If the transmission rate is decreased, max_tr is set again to one. On the other hand, min_suc is increased by 40 when after increasing the transmission rate no single success is achieved and the transmission rate is consequently reduced. If at least one success is achieved at the current rate, min_suc is set again to 20 .

\section{ADAPTIVE S-ALOHA CDMA FOR VOICE AND DATA MulTiPLEXING}

One of the claimed advantages of CDMA is that the randomaccess operating mode of packet CDMA provides near-perfect statistical multiplexing of any given mix of traffic sources. No complex protocol structures are required in the MAC layer for the integration of circuit-mode and packet-mode traffic. This section validates that the proposed adaptive S-ALOHA CDMA scheme is able to handle both real-time and nonreal-time traffic, e.g., voice and data sources, thus satisfying one of the major requirements for any third-generation system. The model presented in Section II can be extended for voice-data transmission by a two-dimensional (2-D) queue model. However, although this model can be analytically formulated, its resolution requires great computational complexity when the number of users is high [22], so we have addressed this situation by using computer simulations.

A classical speech model is considered, where sources create a pattern of talkspurts and gaps, which are assumed to have

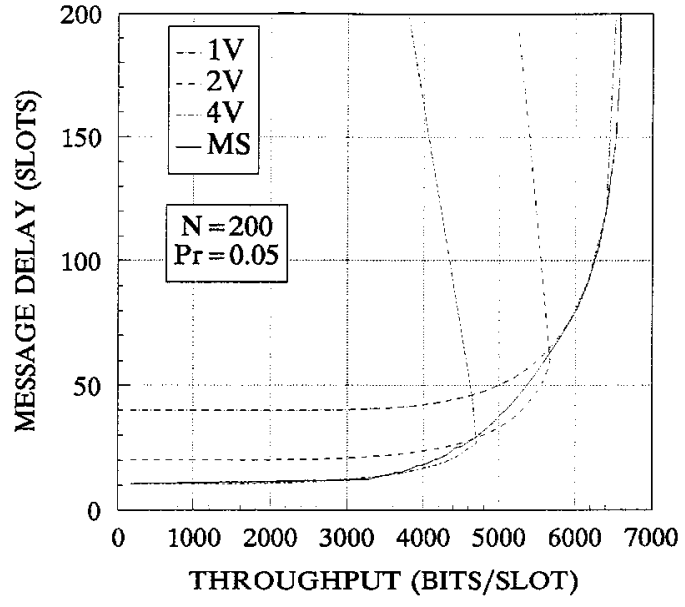

Fig. 5. Performance attained with the MS algorithm.

statistically independent exponentially distributed duration. The mean duration of the talkspurts and gaps are 1.41 and $1.74 \mathrm{~s}$, respectively. This yields a voice activity factor of 0.45 . An 8-Kbps voice encoder is considered. Although S-ALOHA provides a natural protection mechanism, i.e., retransmission, the voice traffic cannot take advantage of it due to the high delay constraints, and so lost packets may occur because voice packets are not retransmitted. Experimental measurements for voice quality indicate that a packet loss of $1 \%$ is still acceptable. In order to diminish the number oflost packets due to multiuser interference, voice information is usually convolutionally encoded. We consider the $r=1 / 2$ convolutional code with generator polynomial (133 171). The following bound for $P_{e}$, the BER after decoding, can be established for this code [23]:

$$
P_{e} \leq \frac{1}{2}\left(36 D^{10}+211 D^{12}+1404 D^{14}+11633 D^{16}\right)
$$

where $D$, for soft decision at the decoder output, is given by

$$
D=e^{-\left(E_{b} / N_{o}\right)} .
$$

Previous results fixed neither the transmission bandwidth nor the time slot duration. At this point, it is necessary to consider some representative values in order to introduce the voice dynamics. Thus, for instance, we could fix a tentative $T_{\text {slot }}=$ $10 \mathrm{~ms}$, which for the reference transmission rate $v \mathrm{~b} / \mathrm{s}(L=$ 200 b) with $G_{p}=127$ leads to a bandwidth occupation $B W=$ $2.54 \mathrm{MHz}$. For a voice encoder delivering $160 \mathrm{~b}$ every $20 \mathrm{~ms}$, we can split this block into two 80-b packets. These $80 \mathrm{~b}$ are convolutionally encoded, giving $160 \mathrm{~b}$, and still leaving room for 40 overhead bits (tail bits for Viterbi decoding and some spare bits in case synchronization is not as ideal as supposed) to complete the 200-b packet.

As a first result, Fig. 6 shows the packet loss rate for a voice-only transmission as a function of the number of voice users $N_{v}$. For a $P_{\text {loss }}=1 \%$ quality threshold, we find $N_{v} \approx 330$ users.

From now onwards, mobiles generating data messages of mean length $(1 / \mu) \mathrm{b}$ and at a rate $\lambda$ are assumed for data simulation purposes. Therefore, the average traffic offered by a single data user is $(\lambda / \mu) \mathrm{b} / \mathrm{s}$. Note that the same average data activity can be caused by highly different message lengths: 


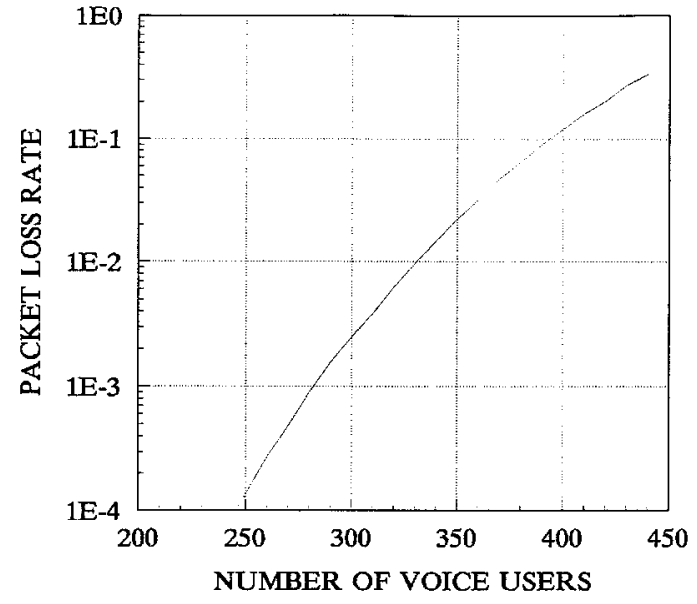

Fig. 6. Packet loss rate for voice-only transmission.

TABLE I

MAXIMUM NUMBERS OF DATA USERS FOR DIFFERENT NUMBERS OF VOICE USERS

\begin{tabular}{l|c|c|c}
\hline$\lambda=0.09 ;(1 / \mu)=1$ Kbyte & $N_{v}=40$ & $N_{v}=60$ & $N_{v}=80$ \\
\hline$v$ bits $/ s$ & 533 & 355 & 182 \\
\hline $2 v$ bits $/ s$ & 395 & 245 & 140 \\
\hline $4 v$ bits $/ s$ & 286 & 164 & 74 \\
\hline
\end{tabular}

from not very frequent, but very long messages to very frequent and very short messages. Every terminal is assumed to have a FIFO buffer with capacity for storing 20 messages. The system is said to be stable while none of the buffers presents overflow. As an additional restriction, the messages should be transferred with the minimum possible delay.

Table I shows the maximum number of data users that can be supported for different numbers of voice users, $N_{v}$, for $\lambda=0.09$ and $(1 / \mu)=1$ Kbyte. In this case $(\lambda / \mu)=720 \mathrm{~b} / \mathrm{s}$, which corresponds to the $20 \%$ of the $3600-\mathrm{b} / \mathrm{s}$ average load generated by a voice user $(8-\mathrm{Kb} / \mathrm{s}$ voice encoder with 0.45 voice activity factor). It is worth noting that increasing the transmission rate by a given factor reduces capacity by less than this factor. Additionally, for higher $\lambda$ a lower number of data users can be supported because of the greater activity. In fact, there is an inverse relation between activity and maximum capacity, as Fig. 7 shows. These results do not include channel coding for data users.

Maximum capacity is not the only pertinent performance measurement, for message delay as a function of the number of registered users also is. Fig. 8 shows the results for data users transmitting at $2 v \mathrm{~b} / \mathrm{s}$ and for the case $\lambda=0.045$ and $(1 / \mu)=1$ Kbyte. Obviously, the lower the number of data or voice users, the lower the minimum message delay will be. As $N_{v}$ increases, the minimum data message delay also increases, since more capacity is devoted to voice transmission.

In the above voice and data integrated framework, the proposed MS algorithm can be applied to data terminals, so the message delivery delay can be reduced when possible (i.e.,

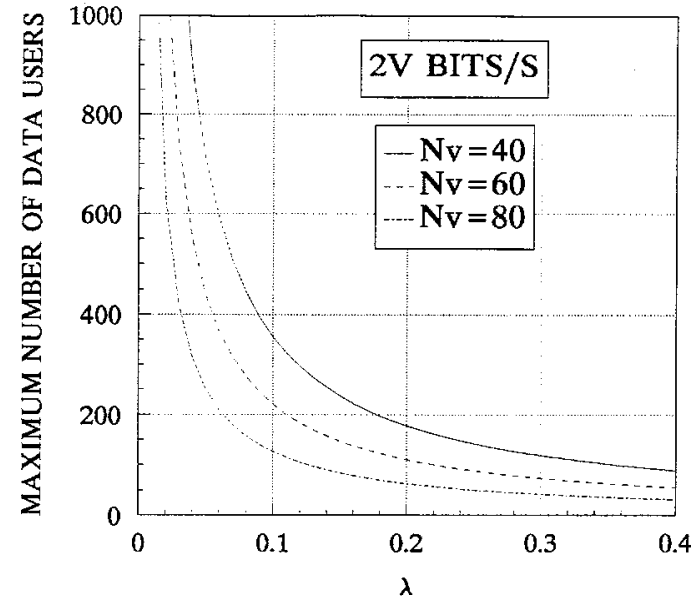

Fig. 7. Impact of data activity on system capacity.

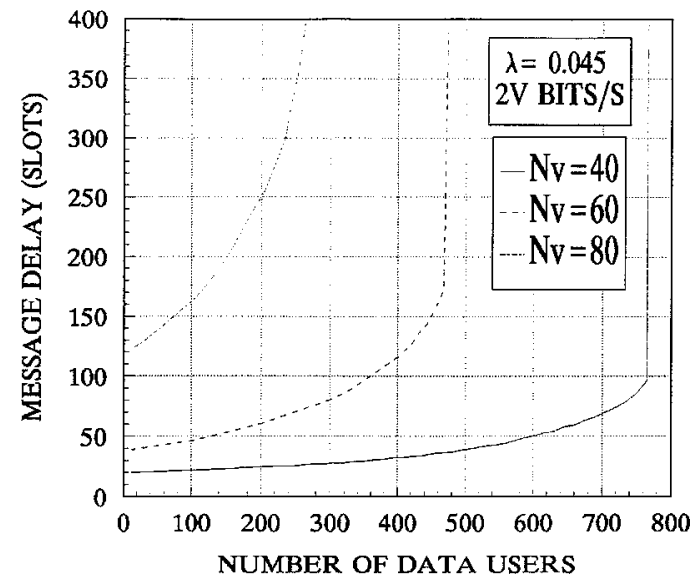

Fig. 8. Data performance for different voice users.

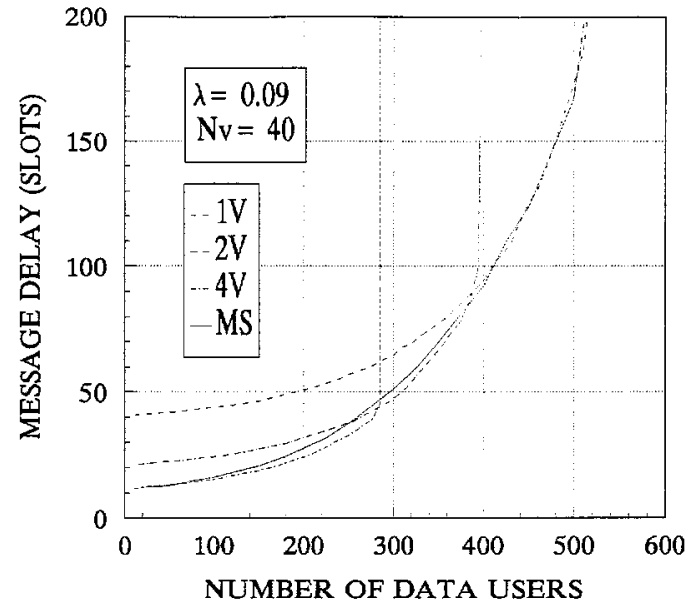

Fig. 9. Data delay behavior with the MS algorithm.

when there is low load in the system) and consequently system efficiency can be improved. Fig. 9 shows the individual performances for data terminals transmitting at different transmission rates and the performance obtained when the MS algorithm is used. We conclude that the MS is able to operate in a mixed voice-data environment, optimizing data transmission in the 
sense that, when possible, data users will try to transmit at higher rates so that the message delay will be reduced.

\section{ADAPTIVE S-ALOHA CDMA IN A CELlular ENVIRONMENT}

When considering multiple-cell CDMA systems, which will be normally the case, the situation becomes more complicated. Basically, the new problem that must be taken into account is the interference level from subscribers in the other cells. This interference varies not only according to the attenuation in the path to the subscriber's cell site, but also inversely to the attenuation from the interfering user to his own cell site, which through power control by that cell site may increase, or decrease, the interference to the desired cell site.

In order to evaluate the MS algorithm in a cellular environment several computer simulations have to be carried out, and the first requirement is to establish a channel model. The propagation attenuation is generally modeled as the product of longand short-term fading. The former is due to the terrain configuration whereas the latter is largely due to multipath reflections. An accepted representation for the received power is

$$
P_{r}=K \times P_{t} \times \frac{\beta^{2}}{r^{\mu} 10^{\zeta / 10}}
$$

where

$P_{r} \quad$ received power;

$P_{t} \quad$ transmitted power;

$\beta^{2}$ exponential random variable representing the Rayleigh fading component;

$\zeta$ decibel attenuation due to shadowing, with zero mean and standard deviation $\sigma$ and the path-loss decays with the $\mu$ th power of distance;

$K$ constant that depends on the gains of the transmitting and receiving antennas and on the frequency.

The long-term fading represents slowly varying variations even for users in motion.

CDMA is able to combat the effect of the fast fading. By using a RAKE receiver and other techniques, the effect of the fast fading can be reduced to a minimum [24]-[26]. Therefore, Rayleigh fading is not considered in our results on the presumption that any fast fading would be effectively combated by the receiver. Thus, as a typical result, the attenuation is affected only by a fourth-power path-loss law and an 8-dB shadow fading. In order to obtain statistics more rapidly, subscribers are located randomly at each time slot and shadowing is also generated independently in a slot by slot basis. Also considered for simulation purposes are a reference cell and its two-tier neighbor cells, so that a total of 18 interfering cells are considered. Mobile terminals are power limited and transmit at most $1 \mathrm{~W}$ (normalized). This condition means that at the edge of the cell site (which is normalized to unity) there is no margin for shadowing fading compensation, and so the instantaneous power control cannot be assured (in the sense that the received power from a subscriber under "bad channel" may be below the desired level). Similarly to [25] and [26], we consider the situation where mobiles are always attached to the lowest attenuation cell site. Under this condition, it holds $I(r) / S \leq 1, I(r)$ being the interference level from a subscriber located at a distance $r$ from the reference cell
TABLE II

DIFFERENT CODING RATES FOR $v$ b/s PACKETS

\begin{tabular}{l|l|l|l}
\hline$k=200$ & $L=267$ & $t=5$ & $r=0.75$ \\
\hline$k=200$ & $L=312$ & $t=10$ & $r=0.64$ \\
\hline$k=200$ & $L=352$ & $t=15$ & $r=0.57$ \\
\hline
\end{tabular}

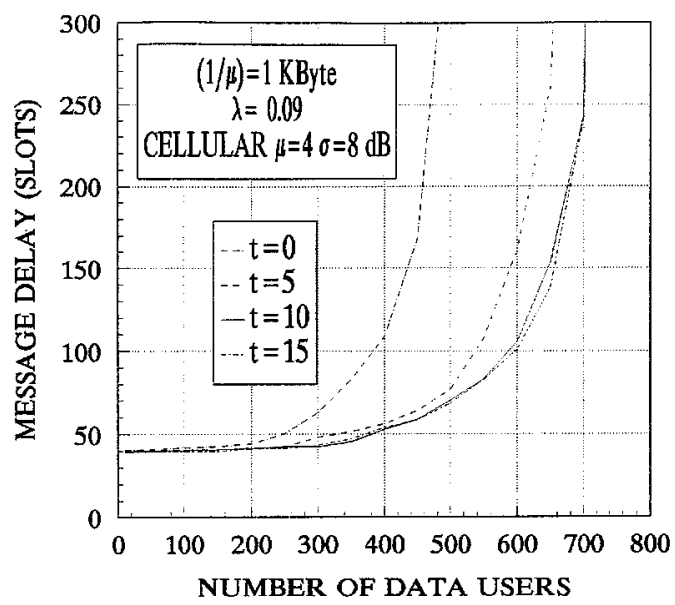

Fig. 10. Data delay performance for different coding rates and fixed transmission rate in a cellular environment.

and $S$ being the desired level at the base station, since otherwise a handover is performed.

Before obtaining performance measurements and given that the interference level is significantly increased when considering the cellular environment, data protection mechanisms (i.e., coding) should be studied in further detail. In order to obtain general results not depending on any specific type of codes, we will use the Varsharmov-Gilbert bound [20], [27], which states that for an $(L, k)$ block code of total length $L \mathrm{~b}$ and $k$ user information bits with capacity for correcting $t$ or fewer errors, the lower bound on the number of information bits $k$ that can be allocated in the block is given by

$$
k \geq L-\log _{2}\left(\sum_{i=1}^{2 t+1}\left(\begin{array}{l}
L \\
i
\end{array}\right)\right) .
$$

Since we are using a spread-spectrum technique, redundancy can be introduced without penalty in terms on bandwidth, but only in processing gain. That is, for a code rate $r=k / L$, the achieved processing gain will be $G_{p}^{\prime}=G_{p} \times r, G_{p}$ being the processing gain when no coding scheme is used and, consequently, $L=k$. We note that the user transmission rate is the same ( $k$ b per time slot) regardless coding is introduced or not. Table II shows different packet configurations and Fig. 10 shows the throughput-delay characteristic for data-only traffic with a fixed transmission rate ( $v \mathrm{~b} / \mathrm{s}, k=200 \mathrm{~b} /$ packet $)$ in a cellular environment. As $t$ is increased a net gain is obtained, denoting that the correction capability introduced at the receiver side outperforms the lower channel processing gain. Moreover, an optimal coding rate is found, in the sense that reducing $r$ beyond a certain value does not led to a performance improvement because a saturation effect arises. In the case of Fig. $10, t \approx 10$ 
TABLE III

OPTIMUM CODING RATES FOR DIFFERENT TRANSMISSION RATES

\begin{tabular}{l|l|l|l|l}
\hline \multicolumn{1}{c}{ bits/s } & $k=200$ & $L=312$ & $t=10$ & $r=0.64$ \\
\hline $2 v$ bits/s & $k=400$ & $L=618$ & $t=20$ & $r=0.647$ \\
\hline 4 bits/s & $k=800$ & $L=1232$ & $t=40$ & $r=0.65$ \\
\hline
\end{tabular}

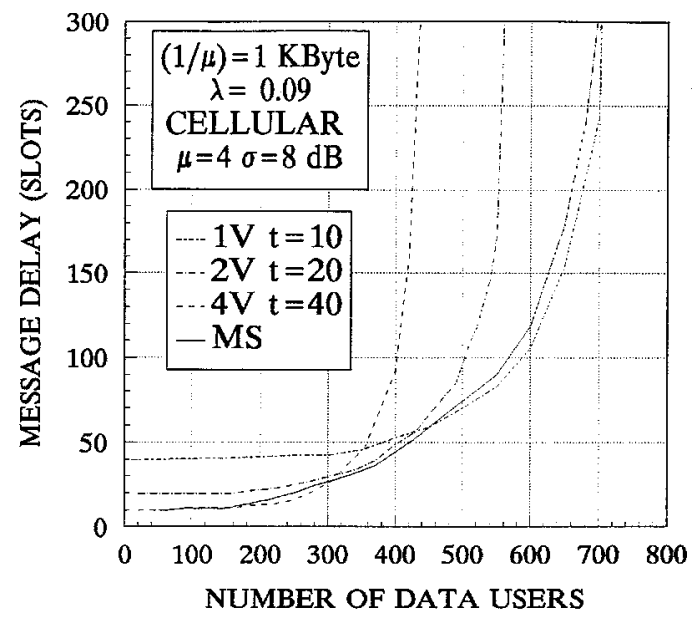

Fig. 11. The MS algorithm performance operating in a cellular environment.

is the optimum value. Similar results are obtained for different transmission rates, so that Table III summarizes the optimum coding rate associated with all three possible transmission rates considered in this paper $(v, 2 v$, and $4 v \mathrm{~b} / \mathrm{s})$.

Coding gain is achieved in addition to the data delay gain deriving from the MS algorithm, so that we fully exploit the CDMA's flexibility in bandwidth utilization by changing the channel transmission rate at two different levels: the first one in order to introduce redundancy while keeping the user transmission rate and the second one by adaptively increasing the users transmission rate when the channel load is low enough. As Fig. 11 shows, the adaptive algorithm performs the same that in the single-cell case, attaining the envelope of the individual rate features, indicating that the change of the transmission rate algorithm is robust to intercell interference. Additionally, Fig. 12 compares the MS performance in a cellular environment with that obtained in a single cell, showing a capacity reduction of about $45 \%$.

Apart from the degradation due to neighbor cell's interference, some other system-related factors may tend to decrease the practical performance as, for example, imperfect power control. Our main interest here is to evaluate the MS algorithm when it is affected by such a problem in order to draw more realistic conclusions. Typically, imperfect power control is modeled by a lognormal distribution affecting the received power. Fig. 13 shows the performance for different values of the standard deviation and a fixed transmission rate $(v \mathrm{~b} / \mathrm{s})$. It can be seen that the degradation due to imperfect power control in terms of maximum number of data users supported is in the range $15 \%$ for $\sigma=1 \mathrm{~dB}$ to $30 \%$ for $\sigma=2 \mathrm{~dB}$. Fig. 14 reveals that the MS al-

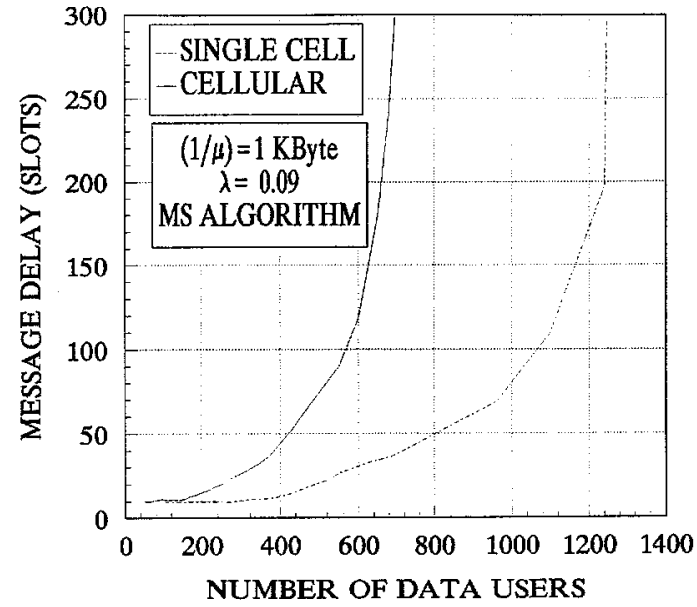

Fig. 12. Comparison between MS algorithm in a single and in a cellular environment.

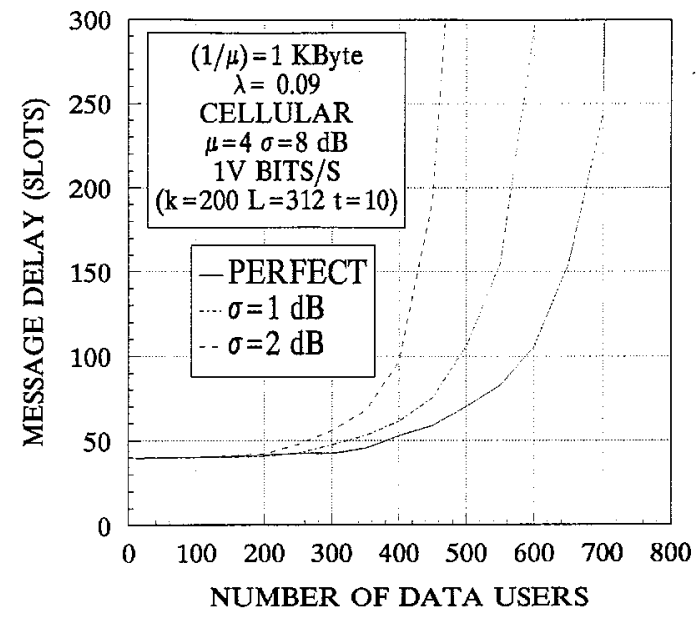

Fig. 13. Degradation due to imperfect power control

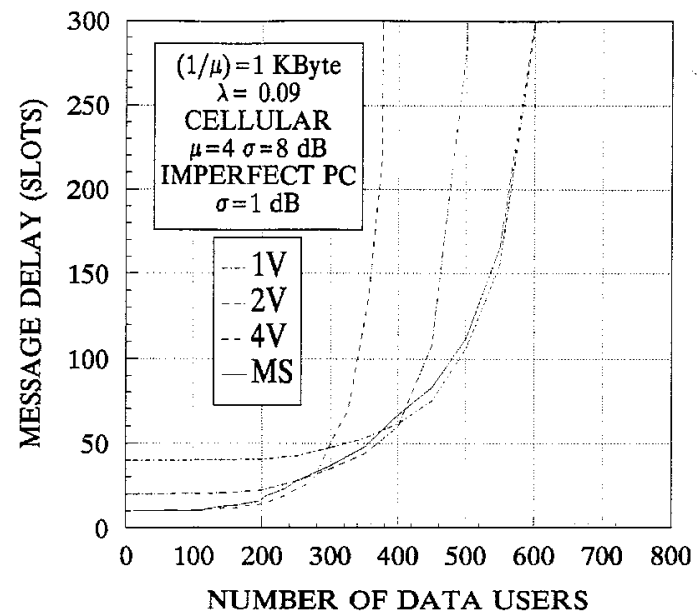

Fig. 14. The MS algorithm performance under imperfect power control.

gorithm also tends to choose the most suitable transmission rate according to the channel load conditions when power control imperfections are present. The performance attained for the MS algorithm with $\sigma=1 \mathrm{~dB}$ lognormal distribution is the envelope of the fixed transmission rate plots. 
DOWNLINK PRMA++ FRAME

\begin{tabular}{|l|l|l|l|l|l|l|l|l|l|l|}
\hline 1 & 2 & 3 & $\mathrm{~A}$ & $\ldots .$. & $\mathrm{FP}$ & 37 & $\mathrm{~A}$ & 39 & $\ldots .$. & 69 \\
\hline
\end{tabular}

\begin{tabular}{|l|l|l|l|l|l|l|l|l|l|l|}
\hline R & 1 & $\ldots .$. & 35 & $\mathrm{R}$ & 36 & 37 & 38 & $\begin{array}{l}\mathrm{FP} \\
\mathrm{ack}\end{array}$ & $\ldots .$. & 69 \\
\hline
\end{tabular}

UPLINK PRMA++ FRAME

Fig. 15. PRMA++ frame structure.

\section{PRMA++ AND ADAPTIVE S-ALOHA DS-CDMA COMPARISON}

Multiple-access techniques and protocols are some of the principal challenges in the design of third-generation mobile radio systems, so that proposing, evaluating, and comparing different solutions have received many interest in recent years. If comparisons try to include the physical layer as well as link layer elements, the model becomes easily untreatable and many mix-up and compensation effects may not allow reaching all conclusions in a very clear way. Therefore, many of the studies concerning the MAC layer simplify the physical layer model or even it is not included at all [8], [28], [29], while those research works covering, for example, power control, sectorization, voice activity monitoring, effects of shadowing and multipath propagation, etc., usually do not introduce any specific MAC protocol, traffic statistics, etc., [30], [31]. The reason is the untreatable models that could come up.

In order to gain more insight into the potentials of the adaptive S-ALOHA CDMA scheme, a comparison with a reservation TDMA-based solution is presented in this section. Since the objective here is to investigate the influence of the new MAC component (i.e., the adaptive change of the transmission rate according to the channel load conditions), the simplified model used is suitable for investigating general system properties, pointing out the key elements favoring both of the protocols considered. However, since the physical layer definitively influences the MAC layer performance, some physical layer issues will be considered to some extent.

The packet-reservation multiple-access (PRMA) protocol, which is an extended version of the R-ALOHA, has been widely studied in recent years. The European research project ATDMA considered another variant, known as PRMA++, as a multiple-access protocol for a third-generation mobile communication system based on the TDMA multiple-access technique [2], [3].

On the uplink of the PRMA++ frame three types of slots exist, as Fig. 15 shows. These are I slots, which are only used for the transmission of information-carrying bursts, $\mathrm{R}$ slots where only access-contention bursts are transmitted, and FP-ack slots used to confirm the correct reception of fast paging bursts on the downlink. The mobile gains access to the channel by transmitting a contention-access burst in an R slot on the uplink frame.
If two or more mobiles transmit access-contention bursts in the same R slot and therefore collide, or if no access-contention attempt is made in an uplink R slot, then the base station transmits an "idle" burst signal via downlink. Hence, mobiles, whose initial access is blocked by collision, back off and recontend in the $\mathrm{R}$ slots at random with a given retransmission probability. When the base station is aware of the mobile access request, it will try to assign an I slot on the uplink frame to that mobile. If there is any I slot available, this channel is reserved for the mobile to transmit its information packets as long as required, until the end of the period of activity, when capacity is released by transmitting an "end of transmission" block.

\section{A. Voice Transmission in an Isolated Cell}

For a fair comparison with PRMA++, the same voice encoder and bandwidth occupancy as in the S-ALOHA CDMA case are retained. As usual in a TDMA scheme, the frame duration is set equal to the voice encoder delivery periodicity, and so a $T_{f}=20 \mathrm{~ms}$ frame is defined. We add 20 overhead bits to the $160-\mathrm{b}$ voice packet generated in this period, forming a 180-b packet. Note that in the case of voice packets for the S-ALOHA CDMA system, 40 overhead bits were introduced, showing that a CDMA system is more complex than a TDMA scheme. For a bandwidth occupation of $B W=R_{b}=\left(1 / T_{b}\right)=2.54 \mathrm{MHz}$, the PRMA++ frame is composed of $R_{b} \times T_{f}=50800 \mathrm{~b}$. Since every slot contains $180 \mathrm{~b}$, we have 282 slots per frame when an isolated cell is considered (the entire bandwidth is available to this cell). Finally, a noiseless nonfading channel is assumed. Therefore, no channel coding is included in the PRMA++ analysis.

As a representative result, we evaluate the PRMA++ protocol with $27 \mathrm{R}$ slots per frame, obtaining a maximum number of voice users of $N_{v} \approx 505$ for a $P_{\text {loss }}=1 \%$. Under these conditions, capacity is far higher than in S-ALOHA CDMA ( $N_{v} \approx 330$, according to Fig. 6).

\section{B. Voice Transmission in a Cellular Environment}

One of the main differences between TDMA and CDMA as multiple-access techniques is that, while the former needs frequency planning to avoid cochannel interference, the latter can bear full frequency reuse. This CDMA property is useful in highly unpredictable environments such as picocells, where effective frequency planning may be impossible to achieve.

For CDMA, voice-only simulations in a cellular environment have been carried out following the model presented in Section VI, i.e., 18 interfering cells, $\mu=4$ and $\sigma=8 \mathrm{~dB}$. For PRMA++ to work properly, a fixed channel allocation (FCA) strategy based on a cell cluster has to be adopted. We assume that cochannel interference can be neglected for a suitable cluster size $K$. Thus, with the same frame duration, the number of slots per frame is reduced by the cell cluster size because less bandwidth per cell is available. For a three-cell cluster, we have 94 slots (one third of the complete 282 slots per frame). For a seven-cell cluster, the number of slots goes down to 40 .

Results are summarized in Table IV, where $N_{v}$ stands for the maximum number of voice users that can be supported with a packet loss rate $\left(P_{\text {loss }}\right)$ below $1 \%$. It can be observed that 
TABLE IV

MAXIMUM CAPACITY FOR VOICE-ONLY TRAFFIC

\begin{tabular}{|c|c|c|c|}
\hline \multicolumn{2}{|c|}{$\begin{array}{l}\text { ACCESS } \\
\text { SCHEME }\end{array}$} & $\begin{array}{c}\text { CELLULAR } \\
\text { ENVIRONMENT }\end{array}$ & $\begin{array}{l}\text { SINCLE } \\
\text { CELL }\end{array}$ \\
\hline \multicolumn{2}{|c|}{ S-ALOHA CDMA } & $N_{v} \approx 160$ & $N_{\nu} \approx 330$ \\
\hline \multirow{3}{*}{ PRMA ++ } & $\begin{array}{l}K=7 \\
\text { (4 R-slots) }\end{array}$ & $N_{v} \approx 67$ & \multirow{3}{*}{$N_{v} \approx 505$} \\
\hline & $\begin{array}{l}\mathrm{K}=4 \\
\text { (7 R-slots) }\end{array}$ & $N_{v} \approx 125$ & \\
\hline & $\begin{array}{l}\mathrm{K}=3 \\
\text { (9 R-slots) }\end{array}$ & $N_{v} \approx 170$ & \\
\hline
\end{tabular}

a)

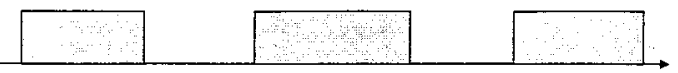

b)

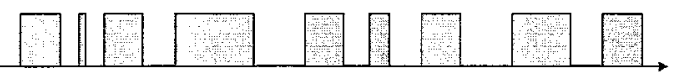

Fig. 16. Different traffic statistics: (a) steady and (b) bursty.

while for the single-cell case, PRMA++ achieves much higher capacity than S-ALOHA CDMA, the situation reverses in a cellular environment, where PRMA++ should be able to operate in a cluster size of only $K=3$ in order to obtain similar figures. For more typical cluster sizes necessary to eliminate cochannel interference $(K=7)$, capacity is considerably reduced. These results show that it is more suitable to use interference-tolerant techniques such as S-ALOHA CDMA rather than "a priori" bandwidth assignment as used in PRMA++.

\section{Voice and Data Multiplexing}

Since the ability to integrate different services is a major requirement for any future system, this issue should also be covered in order to devise a complete access scheme comparison. In what adaptive S-ALOHA CDMA concerns, Section V has partly dealt with this issue. For PRMA++, some additional studies need to be carried out. PRMA++ can transmit data in a similar way to voice information. If any kind of priority for voice over data information is required, the access of the two kinds of traffic to the R slots may differ. With the arrival of an information burst, voice users will occupy the first $\mathrm{R}$ slot available with probability 1 , whereas data users will occupy it with probability $C_{p}$. Thus, voice users can get reservation priority over data users. Note that $C_{p}$ is a protocol parameter that should be determined.

As is common in families of reservation protocols, one could expect PRMA++ to be more sensitive to traffic statistics than S-ALOHA CDMA, which is able to naturally integrate different kinds of traffic sources. Let us consider the situation plot in Fig. 16. Although in both cases the offered load $G$ is the same $(G \approx 1 / 2)$, the higher burstiness of traffic in case b) of Fig. 16 makes it less suitable for PRMA++ operations, since mobile terminals should continuously ask for a reserved channel, and once

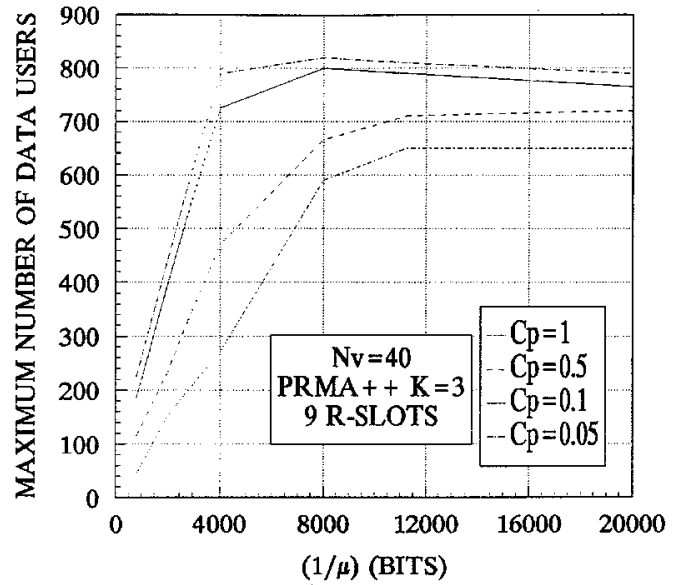

Fig. 17. PRMA++ performance for different traffic sources.

the channel is reserved, the channel would be used for a short period of time.

Several computer simulations for different message lengths have been performed. In all cases, the message arrival rate $\lambda$ was adjusted so that the average data load was $(\lambda / \mu)=720$ $\mathrm{b} / \mathrm{s}$, i.e., $20 \%$ of the average voice load. As an upper bound result, three-cell cluster was assumed for PRMA++, so that the PRMA++ frame contained 94 slots.

Fig. 17 shows that for messages shorter than 1 Kbyte, PRMA++ efficiency drops dramatically for a given frame design. It can also be seen that the lower the $C_{p}$, the higher the maximum data capacity will be. This higher capacity for lower $C_{p}$ is at the expense of higher message delay because the time elapsed for achieving data reservation increases as $C_{p}$ decreases. Moreover, we can conclude from Fig. 17 that a correct operation for PRMA++ requires handling messages at least 1 Kbyte long, otherwise, capacity is severely reduced. This confirms that PRMA++ is designed for voice transmission (a voice talkspurt generates $11280 \mathrm{~b}$ on average) and that this choice may reduce its ability to multiplex other kinds of information sources. Therefore, PRMA++ may be far from being the best choice for supporting multimedia services if some of them are very bursty. These applications have recently been seen as a problem for UMTS because high burstiness may collapse the system if its access mechanism is not flexible enough.

From now on we will assume $C_{p}=0.1$ because this value provides certain priority to voice users, can achieve higher capacity, and finally, the penalty in terms of delay (not shown for the sake of brevity) is relatively small when compared to the minimum data delay $\left(C_{p}=1\right)$.

1) PRMA++ Frame Dimensioning: All previous results in a cellular environment were obtained with nine $\mathrm{R}$ slots in a frame with a total of 94 slots (three-cell cluster). As the number of $\mathrm{R}$ slots per frame has a strong impact on system performance, this parameter should be studied in greater detail. The longer the messages handled, the fewer $\mathrm{R}$ slots should be required and vice versa if messages are short. This demonstrates that is not possible to optimize in all directions and that the system should eventually be designed for a specific application (voice). 


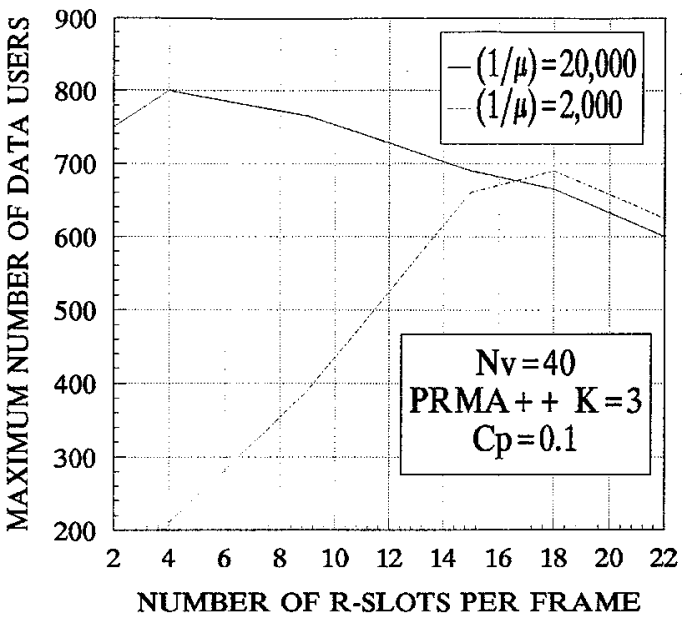

Fig. 18. PRMA++ sensitivity to frame dimensioning.

TABLE V

CAPACITY AND DELAY COMPARISON FOR VOICE AND DATA INTEGRATION

\begin{tabular}{|c|c|c|c|c|c|}
\hline \multirow{3}{*}{$\begin{array}{l}\mathrm{CAPACHY} \\
\left(\mathrm{DEL} \mathrm{AY}_{-\mathrm{S}}\right) \\
\mathrm{N}, 40\end{array}$} & \multicolumn{4}{|c|}{ W } & \multirow{3}{*}{ S Adaptre } \\
\hline & \multicolumn{2}{|c|}{$\mathrm{K}=3$} & \multicolumn{2}{|c|}{$\mathrm{K}=7$} & \\
\hline & $4 \mathrm{R}$-slot & $18 \mathrm{R}$ Slot & $2 \mathrm{R}$ slot & $7 \mathrm{R}$-slot & \\
\hline$(1 / \mu)=2000$ & $\begin{array}{c}210 \\
(0.24-0.34)\end{array}$ & $\begin{array}{c}690 \\
0.22-0.29)\end{array}$ & $\begin{array}{c}80 \\
0.26-0.39\end{array}$ & $\begin{array}{c}280 \\
(0.24-0.36\end{array}$ & $\begin{array}{c}550 \\
(0.03-0.18)\end{array}$ \\
\hline 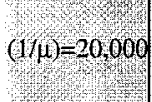 & $\begin{array}{c}800 \\
(2.26-2.36\end{array}$ & $\begin{array}{c}665 \\
2.24-2.29\end{array}$ & $\begin{array}{c}330 \\
2.30-2.87\end{array}$ & $\begin{array}{c}260 \\
(2.27-2.72\end{array}$ & $\begin{array}{c}560 \\
(0.26-0.48)\end{array}$ \\
\hline
\end{tabular}

Fig. 18 presents the capacity for long [e.g., $(1 / \mu)=20000 \mathrm{~b}$ ] and short [e.g., $(1 / \mu)=2000 \mathrm{~b}$ ] messages as a function of the number of R slots per frame. We find that for $(1 / \mu)=2000$, data capacity is maximized with $18 \mathrm{R}$ slots and for $(1 / \mu)=$ 20000 , the best choice is four $\mathrm{R}$ slots. If the final dimensioning is four $\mathrm{R}$ slots per frame, the ability to multiplex short messages drops quite rapidly. On the other hand, if the PRMA++ is optimized for bursty traffic, the ability to support long messages decreases, although not so fast as in the previous case. For any other frame dimension, similar procedure should be carried out in order to find the optimum number of $\mathrm{R}$ slots per frame.

2) Comparison Between PRMA++ and Adaptive S-ALOHA CDMA: Table V summarizes the voice-data integration ability for PRMA++ as well as adaptive S-ALOHA CDMA. For the sake of brevity, only the maximum capacity and, in brackets, the message delay intervals (i.e., the minimum delay-few active users - and the maximum delay - heavy load) are represented. Similarly to Table IV, where only voice traffic was considered, in order to obtain similar capacity figures, PRMA++ should be able to operate in only a three-cell cluster configuration. For more typical cluster values, as for example 7 , the capacity is significantly lower than that provided by adaptive S-ALOHA CDMA. Moreover, in terms of message delay we find a clear superiority of S-ALOHA CDMA with MS algorithm over PRMA++: there is a dramatic decrease in data delay with adaptive S-ALOHA CDMA compared to PRMA++. TDMAbased access protocols may also reduce data delay by assigning multiple slots per frame, but this requires a scheduling algorithm at the base station. However, the criteria to be used at this scheduler are not clear because they are highly dependent on the specific applications. In fact, there are few studies of this topic reported in the literature because it is difficult to establish general criteria in a world where applications and requirement are ever evolving. The growth of Internet-based applications is just one example of how the natural multiplexing ability of CDMA may make it a suitable basis for third-generation systems.

\section{CONCLUSIONS}

In this paper, we have proposed an adaptive S-ALOHA CDMA access technique as a strategy for coping with the requirements of both bursty-packet and voice transmission operating in a multicell scenario. The solution is based on CDMA's flexibility in bandwidth utilization, allowing the change of the transmission rate when for any reason the network is operating below maximum capacity. By means of analysis and simulations, the capability to integrate different traffic sources while providing close to the optimum data delay has been found. Additionally, mobile terminals also choose the most suitable transmission rate when operating in a cellular environment, where intercell interference plays a role and even when power control imperfections are present. Finally, the advantages claimed for the proposed noncentralized scheme have also been compared to those obtained with the centralized PRMA++ in a simplified multicell model, showing the suitability of adaptive S-ALOHA CDMA, mainly because of the simplicity in fully exploiting the time-varying channel load conditions.

\section{REFERENCES}

[1] S. Nanda, D. J. Goodman, and U. Timor, "Performance of PRMA: A packet voice protocol for cellular systems," IEEE Trans. Veh. Technol., vol. 40, pp. 584-598, Aug. 1991.

[2] J. Devile and J. Dunlop, "Development of the multiple access technique for the ATDMA air interface," in RACE Mobile Workshop, June 16-18, 1993, pp. 425-429.

[3] G. Anastasi, D. Grillo, and L. Lenzini, "An access protocol for speech/data/video integration in TDMA-based advanced mobile systems," IEEE J. Select. Areas Commun., vol. 15, pp. 1498-1510, Oct. 1997.

[4] I. Katzela and M. Naghshineh, "Channel assignment schemes for cellular mobile telecommunication systems: A comprehensive survey," IEEE Personal Commun., vol. 3, pp. 10-31, June 1996.

[5] "A guide to DECT features that influence the traffic capacity and the maintenance of high radio link transmission quality, including results of simulations," ETSI Doc. RES(92)09 Annex 4.

[6] N. Abramson, "Multiple access in wireless digital networks," Proc. IEEE, vol. 82, pp. 1360-1369, Sept. 1994.

[7] M. Zorzi and S. Pupolin, "Slotted ALOHA for high-capacity voice cellular communications," IEEE Trans. Veh. Technol., vol. 43, pp. 1011-1021, Nov. 1994.

[8] Z. Liu and M. El Zarki, "Performance analysis of DS-CDMA with slotted ALOHA random access for packet PCNs," Wireless Networks, pp. 1-16, 1995.

[9] D. Raychaudhuri, "Performance analysis of random access packet-switched code division multiple access systems," IEEE Trans. Commun., vol. COM-29, pp. 895-901, June 1981.

[10] A. Polydoros and J. Silvester, "Slotted random access spread-spectrum networks: An analytical framework," IEEE J. Select. Areas Commun., vol. SAC-5, pp. 989-1002, July 1987. 
[11] A. Salmasi and K. S. Gilhousen, "On the system design aspects of CDMA applied to digital cellular and personal communications networks," in Proc. 41st IEEE Veh. Technol. Conf., St. Louis, MO, May 1991.

[12] R. Ganesh et al., "Performance of cellular packet CDMA in an integrated voice/data network," Int. J. Wireless Inform. Networks, vol. 1, no. 3, pp. 199-222, 1994.

[13] N. Guo, S. D. Morgera, and P. Mermelstein, "Common packet data channel (CDPC) for integrated wireless DS-CDMA network," IEEE J. Select. Areas Commun., vol. 14, pp. 735-748, May 1996.

[14] L. Tan and Q. T. Zhang, "A reservation random-access protocol for voice/data integrated spread-spectrum multiple-access system," IEEE J. Select. Areas Commun., vol. 14, pp. 1717-1727, Dec. 1996.

[15] O. Sallent and R. Agustí, "A proposal for an adaptive S-ALOHA access system for a mobile CDMA environment," IEEE Trans. Veh. Technol., vol. 47, pp. 1019-1023, Aug. 1998.

[16] - "Adaptive schemes for packet data in a DS-CDMA environment," in Proc. IEEE 47th Veh. Technol. Conf., VTC'97, pp. 1019-1023.

[17] E. Dahlman et al., "AUMTS/IMT-2000 based on wideband CDMA," IEEE Commun. Mag., vol. 36, pp. 70-80, Sept. 1998.

[18] F. Adachi et al., "Wideband DS-CDMA for next-generation mobile communications systems," IEEE Commun. Mag., vol. 36, pp. 56-69, Sept. 1998.

[19] M. B. Pursley, "Performance evaluation for phase-coded spread-spectrum multiple-access communication-Part I: System analysis," IEEE Trans. Commun., vol. COM-25, pp. 795-799, Aug. 1977.

[20] R. K. Morrow and J. S. Lehnert, "Packet throughput in slotted ALOHA DS/SSMA radio systems with random signature sequences," IEEE Trans. Commun., vol. 40, pp. 1223-1230, July 1992

[21] L. Kleinrock, Queueing Systems. New York: Wiley, 1975.

[22] J. M. Jacobsmeyer, "Congestion relief on power-controlled CDMA networks," IEEE J. Select. Areas Commun., vol. 14, pp. 1758-1761, Dec. 1996.

[23] M. K. Simon, J. K. Omura, R. A. Scholtz, and B. K. Levitt, Spread Spectrum Communications. Rockville, MD: Computer Science, 1985.

[24] C.-C. Lee and R. Steele, "Effect of soft and softer handoffs on CDMA system capacity," IEEE Trans. Veh. Technol., vol. 47, pp. 830-841, Aug. 1998.

[25] K. S. Gilhousen et al., "On the capacity of a cellular CDMA system," IEEE Trans. Veh. Technol., vol. 40, pp. 303-312, May 1991.

[26] A. J. Viterbi et al., "Soft handoff extends CDMA cell coverage and increases reverse link capacity," IEEE J. Select. Areas Commun., vol. 12 pp. 1281-1287, Oct. 1994.

[27] S. Lin and D. J. Costello, Error Control Coding: Fundamentals and Applications. Englewood Cliffs, NJ: Prentice-Hall, 1983.
[28] A. E. Brand and A. H. Aghvami, "Performance of a joint CDMA/PRMA protocol for mixed voice/data transmission for third generation mobile communication," IEEE J. Select. Areas Commun., vol. 14, pp. 1698-1707, Dec. 1996.

[29] T. Papantoni-Kazakos, N. B. Likhanov, and B. S. Tsybakov, "A protocol for random multiple access of packets with mixed priorities in wireless networks," IEEE J. Select. Areas Commun., vol. 13, pp. 1324-1331, Sept. 1995.

[30] F. D. Priscolli and F. Sestini, "Effects of imperfect power control and user mobility on a CDMA cellular network," IEEE J. Select. Areas Commun., vol. 14, pp. 1809-1817, Dec. 1996.

[31] M. G. Jansen and R. Prasad, "Capacity, throughput and delay analysis of a cellular DS-CDMA system with imperfect power control and imperfect sectorization," IEEE Trans. Veh. Technol., vol. 44, pp. 67-75, Feb. 1995.

Oriol Sallent (M'98) received the Engineer and Doctor Engineer degrees in telecommunication from the Universitat Politècnica de Catalunya (UPC), Barcelona, Spain, in 1994 and 1997, respectively.

He joined the Escola Tècnica Superior d'Enginyeria de Telecomunicació de Barcelona, Barcelona, where he became an Assistant Professor in 1994 and an Associate Professor in 1998. His research interests are in the field of mobile communication systems, especially packet radio techniques and spread-spectrum systems.

Dr. Sallent received the Doctorate Award from the Telecommunication Engineer Association of Spain in 1997 for his Ph.D. degree dissertation on multiple-access protocols for CDMA-based systems.

Ramon Agustí (M'78) was born in Riba-roja d'Ebre, Spain, on August 15, 1951. He received the Engineer of Telecommunications degree from the Universidad Politécnica de Madrid, Madrid, Spain, in 1973 and the Ph.D. degree from the Universitat Politècnica de Catalunya, Barcelona, Spain, in 1978.

In 1973, he joined the Escola Tècnica Superior d'Enginyers de Telecomunicació de Barcelona, Barcelona, where he became a Full Professor in 1987. $\mathrm{He}$ has been working in the field of digital communications with particular emphasis on digital radio, both fixed radio relay and mobile communications. $\mathrm{He}$ has also been concerned with the performance analysis and development of frequency-hopped spread-spectrum systems. He participated in the COST 231, RACE, and ACTS European Research Programs and currently is participating in the IST program. His research interest is in the area of mobile communications with special emphasis on CDMA systems and packet radio networks. 